\title{
Secolarizzazione e fattore religioso: una nuova convivenza
}

\author{
Secularization and Religious Factor: a new coexistence
}

\author{
Stelio MANGIAMELI \\ Università degli Studi di Teramo (Italia) \\ mangiameli@dsgpub.giur.unite.it
}

Riassunto: Il testo prende le mosse dall'esposizione delle principali tappe del processo storico che ha portato alla definitiva distinzione tra autorità religiosa e autorità mondana in Europa. Dopo aver identificato il fondamento della politica nella la libertà della Chiesa; ed il fondamento dello Stato nella libertà religiosa; I'Autore giunge a due ordini di conclusioni: la prima è che il cristianesimo ha giocato un ruolo fondamentale nell'avvio e nello sviluppo della secolarizzazione. La seconda, che a suo avviso merita di essere chiarita e messa alla prova dei tempi, consiste nella pretesa di relegare lo Stato e la politica negli angusti confini della mondanità, ovvero, nella pretesa di escludere che Stato e politica abbiano implicazioni spirituali. Egli intende quindi mettere alla prova l'idea che lo Stato liberale di diritto possa resistere, a scapito delle molte lacerazioni che ne frammentano il corpo sociale, senza operare un riferimento a princìpi e valori ad esso paradossalmente estranei. Considerando il problema sotto questa specifica prospettiva, appare chiaro come due concetti affatto estranei alla tradizione europea, quali il principio di tolleranza ed un «sana laicità», possano rappresentare la chiave di volta che ci consenta di superare le contraddizioni implicite e le tensioni irrisolte che ancora oggi attraversano la nostra società post-moderna e secolarizzata.

Parole chiave: libertà religiosa; principio di laicità; principio di tolleranza; secolarizzazione; Stato Liberale di Diritto.
Abstract: The paper begins displaying the main stages of the historical process that has led to the definitive distinction between religious authority and civil authority in Europe. After identifying the foundation of politics in the freedom of the Church; and the foundation of the State in religious freedom; the Author comes to two conclusions: the first is that Christianity played a key role in the birth and development of secularization. The second, which in his opinion deserves to be clarified and put to the test of the times, consists in the claim of relegating State and politics in the narrow limits of worldliness. The claim of excluding that State and politics have spiritual implications. He therefore intends to test the idea that the liberal state of law could resist, at the expense of the many lacerations that fragment its social body, without the help of principles and values which it could not justify. Considering the problem under this particular perspective, it is clear that two concepts that characterize the European tradition, such as the principle of tolerance and an «healthy secularism», could represent the keystone that allows us to overcome the implicit contradictions and the unresolved tensions which still cross our post-modern and secular society.

Keywords: religious freedom; secularism; tolerance; secularization; Liberal State of Law 


\section{L'AUTONOMIA DELLA POLITICA E DELLO STATO COME FRATTURA DALLA RELIGIONE E DALLA CHIESA}

U n tema fondamentale della storia europea è la distinzione e la separazione tra «spirituale»e $\ll$ mondano» 1 .

Chi volesse ripercorrere a ritroso l'intera vicenda della dialettica tra queste due categorie, dovrebbe tornare indietro sino alla lotta per le investiture e alla celebre umiliazione di Canossa (gennaio del 1077) dell'imperatore Enrico IV, davanti al Papa Gregorio VII².

Non è vano ricordare che in gioco vi era la libertà della Chiesa (libertas Ecclesiae), nei confronti dell'uso sconsiderato degli uomini, sia pure imperatori, di rafforzare il loro potere piegando la sfera spirituale al loro servizio. La religione cristiana di quel periodo esprimeva un vincolo politico e giuridico di cui si avvantaggiava il potere politico e non esisteva né la libertà religiosa, sia pure come libertà della coscienza, né la possibilità di distinguere tra foro esterno e foro interno.

Ora, nonostante i comportamenti successivi dell'imperatore «finto pentito», lo sconfitto di quella vicenda fu proprio Enrico IV, al quale il perdono papale non aveva restituito alcun potere spirituale e la cui persona, nella carica politica, era stata per sempre desacralizzata. Non più sovrano unto con l'olio santo nel nome di Dio, che ne faceva un consacrato al pari del Papa, sia pure per un ufficio diverso, bensì semplice uomo che aveva concentrato in sé il potere temporale, in maniera più o meno legittima.

Ma con molta probabilità Enrico IV teneva in poco conto la propria sacralità e, senza la dovuta consapevolezza, la sua brama di potere - inteso questo nel senso più angusto del termine, come capacità di dominio su altri uomini asserviti alla propria volontà - fonda la sfera della politica, che non è più il risvolto della religione, ma un campo dell'agire umano, a sé stante, dove si svolge la lotta tra gli uomini per la supremazia. Il fondamento della politica non è spirituale e così come desacralizzata è la persona dell'imperatore, desacralizzata è pure la politica. La religione parla di Dio, la politica parla degli uomini, la prima serve allo spirito, la seconda alla vita terrena ${ }^{3}$.

1 Sull'impossibilità di conciliare spiritualità e mondanità v. DE LuBAC, H., Meditazioni sulla Chiesa (1953), Jaca Book, 2008.

2 Vid. WAHL, R., Der Gang nach Canossa- Kaiser Heinrich IV, Verlag F. Bruckmann, München, 1952; SChNeIdmüller, B.; WeInfurter, S. (Hrsg.), Die deutschen Herrscher des Mittelalters, Historische Porträts von Heinrich I. bis Maximilian I. (919-1519), C. H. Beck, Monaco, 2003.

3 Vid. Locke, J., Lettera sulla tolleranza (1689), a cura di C. A. Viano, Laterza, Roma-Bari, 2005. 
Questo l'inizio del processo di secolarizzazione nel cui orizzonte alcuni secoli dopo si inserisce lo Stato, inteso non come entità metafisica e spirituale, ma come strumento politico storicamente determinato e ubbidiente alla logica di un ben diverso conflitto religioso ${ }^{4}$.

Tutto comincia con la riforma (1517) e con la divisione che essa introduce tra i cristiani, dopo lo scisma d'oriente (1054), in quella parte dell'impero che per semplicità possiamo chiamare «Europa».

La riforma, che oggi sembra un accadimento storico quasi innocuo, in realtà comportò una trasformazione profonda dell'equilibrio europeo attraverso le guerre di religione, guerre crudeli che causarono morte e povertà.

L'esito di quella vicenda, alla fine della guerra dei trent'anni, fu la pace di Westfalia (1648), i cui risultati si possono riassumere in due elementi essenziali. Da un lato, la cessazione di pretese religiose, verso i sudditi, da parte dei principi che avevano il potere territoriale; dall'altro, la formazione del pluralismo degli ordinamenti politici territoriali, all'interno di una comunità pacificata dal punto di vista religioso e divisa, semmai, dalle lotte di potere.

Con l'assetto di Westafalia si assiste alla perdita di significato politico delle autorità universali: Impero e Papato; e il principio cardine di questa, ampiamente noto, cuius regio eius religio, istituzionalizza la secolarizzazione ${ }^{5}$. Lo Stato, l'ordinamento politico territoriale, è la nuova organizzazione del potere pubblico. Lo Stato, come sfera istituzionale, è integralmente laico, privo cioè di qualunque fondamento spirituale e il suo potere è mondano con obiettivi tutti materiali e terreni; esso è forte per la concentrazione della forza che realizza e il monopolio nella creazione del diritto che determina; mette fuori gioco tutti i legami giuridici e sociali che definivano la posizione dell'individuo come parte di una o più comunità: la chiesa, la corporazione, la città, il feudo, il clan, la famiglia, ecc.

Così come la riforma aveva posto il credente davanti a Dio, in una alterità spirituale completamente incommensurabile, la formazione dello Stato pone l'individuo davanti al Sovrano, anche in questo caso in una alterità incommensurabile sia pure di potere terreno ${ }^{6}$. Lo schema strutturale del potere politico

4 Tra coloro che considerano lo Stato come un portato della secolarizzazione vid. SCHMITT, C., Teologia politica: quatto capitoli sulla dottrina della sovranità (1933), trad. it. in Le categorie del politico, a cura di G. Miglio e P. Schiera, il Mulino, Bologna, 1972, 29 ss.

5 Vid. BöCKENFÖRDE, E. W., La nascita dello Stato come processo di secolarizzazione, trad. it. in Diritto e secolarizzazione. Dallo Stato moderno all'Europa unita, a cura di G. Preterossi, Laterza, Bari, 2008, 33 ss.

6 Vid. KAISER, J.H., La rappresentanza degli interessi organizzati, trad. it., a cura di S. Mangiameli, Giuffrè, Milano, 1993. 
è quello dell'assolutezza religiosa, che è propria dell'istituzionalizzazione delle Chiese; ma, nel caso dello Stato in una dimensione mondana, in cui la forza può sottomettere persino la Chiesa, o può appropriarsi di questa, come nel caso inglese, senza mai raggiungere, però, l'assolutezza spirituale.

Anzi, l'istituzione dello Stato, con fini puramente terreni, muove dal riconoscimento che questa istituzione non è competente nella materia spirituale e che l'individuo purché mantenga la sua fedeltà all'istituzione e cioè soggiaccia al potere assoluto del sovrano come suddito e popolo, è libero di credere in ciò che vuole e per il suo credo non può essere sottoposto a forme di discriminazione giuridiche. Si formano così due sfere distinte: quella istituzionale (lo Stato) nella quale conta solo il legame di subordinazione al sovrano, che riguarda il comportamento esterno del suddito; e quella sociale nella quale l'individuo e i gruppi sono lasciati liberi di professare la loro religione e, in questo modo, di salvaguardare la sfera interiore che non subisce più le costrizioni del passato.

In definitiva, la libertà della Chiesa fonda la politica, la libertà religiosa fonda lo Stato ${ }^{7}$ entrambe politica e Stato non poggiano sulla dimensione spirituale e sulla teologia, anche se da questa ricavano schemi logici e modelli di comportamento che possono consentire loro di strutturarsi, ma necessitano di essere giustificati e fondati: la politica trova giustificazione e fondazione nella ragione della natura umana, lo Zoon politikon di Aristotele, che sottolinea l'essenza politica dell'uomo, che si determina nei fini da perseguire per mezzo della recta ratio; lo Stato è fondato e giustificato - secondo l'insegnamento di Hobbes - dai quattro compiti che il sovrano deve assolvere in favore del suo popolo e per giustificare l'esercizio del suo potere, e cioè: 1 . che il popolo sia difeso contro i nemici esterni; 2 . che la pace interna sia preservata; 3 . che sia possibile arricchirsi così tanto, come è possibile, godendo della pubblica sicurezza; 4. che goda di una libertà innocente ${ }^{8}$.

Per i sovrani non può esservi maggiore felicità civile, di essere preservati dalle guerre estere e civili, e nell'adempiere al proprio compito il loro com-

7 A tal riguardo, oltre a LOCKE, op. cit., vid. PUfENDORf, S., Über die Natur und Eigenschaft der christlichen Religion und Kirche in Ansehung des bürgerlichen Lebens und Staats (1687), e per un esame del pensiero di questo cfr.: PalladinI, F., «Stato, chiesa e tolleranza nel pensiero di S. Pufendorf», in Rivista storica italiana (1997), 436-482. Riferimenti anche nei principi della dichiarazione di indipendenza, prima, e nel I emendamento alla Costituzione, vid. peraltro JEFFERSON, Th., A Bill for Establishing Religious Freedom (1779).

8 Vid. Hobbes, Th., De Cive (1642), XIII, VI, trad. it. a cura di N. Bobbio, Utet, Torino, 1948, 273. 
penso è di godere tranquillamente della ricchezza che hanno acquistato con la propria operosità.

Il sovrano in questa istituzione originale che è lo Stato esprime l'intera sfera politica, ciò gli consente di non ubbidire alle c.d. leggi, ma alla sola legge suprema che è la sicurezza del popolo ed è guidato dall'obbedienza alla recta ratio.

La sua persona costituisce l'identità dello Stato. La sua sovranità è territoriale; la comune soggezione degli individui alla sua sovranità fa della moltitudine dei sudditi un popolo. Lo Stato è governato per mezzo delle istituzioni e delle leggi che il sovrano pone. Le leggi sono poste dal suo potere che rende assoluta la sua volontà che è volontà cui si deve, perciò, obbedienza assoluta, con l'eccezione del diritto di resistenza posto a salvaguardia, della vita stessa del suddito?.

Anche se lo stesso Hobbes, nel definire la recta ratio, parla della «legge naturale, morale e divina», non la considera in modo oggettivo e aristotelico, bensì in maniera soggettiva come volontà sovrana ${ }^{10}$.

Occorre a questo punto fornire due precisazioni.

In primo luogo, va ricordato che il processo storico della secolarizzazione della politica e dell'istituzionalizzazione del potere secolare nello Stato non è stato lineare e senza contraddizioni, ma lungo ed intriso di aporie e inframmettenze: la religione cattolica, prima, e quella cristiana, poi, hanno rappresentato pur sempre il comune denominatore del continente europeo; anche dopo Westfalia i principi hanno continuato a farsi ungere con l'olio santo e a nominare vescovi e a richiamarsi alla legge divina per giustificare decisioni politiche spesso nefande e, persino, a decidere quale fosse la vera religione ${ }^{11}$.

Insomma, l'avvio e lo sviluppo della secolarizzazione hanno operato in collegamento con la religione e in contesto ben lungi dall'essere scristianizzato.

In secondo luogo, la frattura emersa con la rivendicazione dell'autonomia della sfera politica dal religioso e l'istituzionalizzazione dell'ordinamento

9 Vid. ZaRKA, Y. Ch., «La mutazione del diritto di resistenza in Grozio e Hobbes. Dal diritto collettivo del popolo al diritto dell'individuo», trad. di G.C., in Rivista di Storia della Filosofia, vol. 50, n 3 (1995), pp. 543-556.

10 Vid. Hobbes, T., De Cive (1642), XIII, II, cit., 270.

11 Per una ricostruzione della secolarizzazione v. LüBbe, H., Säkularisierung. Geschichte eines ideenpolitischen Begriffs, K. Alber, Freiburg-München, 1965 (tr. it.: La secolarizzazione. Storia e analisi di un concetto, il Mulino, Bologna, 1970). 
politico territoriale nello Stato, che stanno alla base del processo di secolarizzazione, avrebbero determinato in via di principio l'irrilevanza della religione dalla politica e la neutralità istituzionale dello Stato nelle questioni religiose ${ }^{12}$. Altrimenti detto: la secolarizzazione avrebbe determinato che Stato e politica sono affari interamente mondani e non hanno implicazioni spirituali.

Prima di considerare se queste affermazioni mantengano la loro significatività anche oggi, o non rappresentino piuttosto delle limitazioni del processo di secolarizzazione, per cui né lo Stato, né la politica possono fare a meno di poggiarsi sulla religione, occorre osservare che per effetto della secolarizzazione la libertà religiosa e le Chiese sono collocate e dovrebbero vivere solo nella sfera sociale e che, da tale posizione, possono solo interloquire con la politica e le istituzioni del potere pubblico, rimanendo interamente soggette all'assolutismo della politica e a quello dello Stato ${ }^{13}$.

Ciò nonostante - e questo è il punto rilevante - anche a considerare corretta questa collocazione limitativa della religione, la frattura su cui poggia la secolarizzazione pone il tema della «convivenza» tra credo e non credo, tra i diversi credi, tra istituzioni pubbliche e istituzioni religiose. La questione - come è noto - ha un suo punto di riferimento storico nell'editto (di Nantes, 1598) di Enrico IV di Navarra con il quale veniva creata una situazione di convivenza territoriale delle fedi che non ne mitigava il reciproco assolutismo, ma che avrebbe impedito che le parti contendenti venissero a confronto con le armi, salvaguardando così la pace ${ }^{14}$. Mentre la situazione sarebbe cambiata effettivamente con l'Editto di tolleranza (Luigi XVI, 1787) ${ }^{15}$, poi con il riconoscimento nella Dichiarazione del 1789 della libertà di religione (art. 10) e quella di opinione (art. 11) ${ }^{16}$ e con la Costituzione del 1791, nel cui titolo I è

12 Sul tema della neutralità v. Brenner, M., Staat und Religion, in VVDStRL, Heft n. 59, De Gruyter, Berlin, 1999, 265 ss.

13 Il tema ancora di recente ha suscitato notevole interesse, v. LuCKMANn, T., The invisible religion. The transformation of symbols in industrial society, MacMillan, New York, 1963 (tr. it.: La religione invisibile, il Mulino, Bologna, 1969); PARSONS, T., «Religion in postindustrial America: the problem of secularization», in Social research (1974), pp. 19-225.

14 Sull'editto di Nantes v. LÉONARD, E.G., Histoire générale du protestantisme, vol. II, L'Etablissement (1564-1700), P.U.F., Paris, 1961, 312-89. L'editto venne revocato, poi, da Luigi XIV con l'Editto di Fontainebleau (18 ottobre 1685), che provocò l'espulsione di circa 300.000 ugonotti.

15 Vid. LeCler, J., Histoire de la tolérance au siècle de la Réforme, Albin Michel, Paris, 1994; MaYEur, J.-M.; Venard, M.; Pietri, L.; Vauchez, A., Les défis de la modernité (1750-1840). Histoire du christianisme, Tome 10, Desclee, Fleurus, 1997, 567.

$16 \mathrm{Vid}$. Art. 10: «Nessuno deve essere molestato per le sue opinioni, anche religiose, purché la manifestazione di esse non turbi l'ordine pubblico stabilito dalla legge». Art. 11: «La libera comu- 
riconosciuta e garantita come diritto naturale e civile «la libertà ad ogni uomo... di esercitare il culto religioso al quale è legato», il diritto dei cittadini «di eleggere o scegliere i ministri dei loro culti» e la messa a disposizione, da parte della Nazione, cui appartengono, «di beni destinati alle spese del culto ${ }^{17}$.

Già su questo terreno l'espressione «laicità» esprime una serie di ambiguità che sono ben note al dibattitto costituzionalistico e che vanno dalla indifferenza dello Stato verso la religione, alla neutralizzazione del fattore religioso come funzione attiva dello Stato, oppure alla garanzia dello Stato per la salvaguardia della libertà di religione (in regime di pluralismo confessionale e culturale $)^{18}$.

Tuttavia, non è questo che rileva al momento, quanto piuttosto la circostanza che la frattura presente nella società e dovuta alla presenza di più fedi,

nicativa dei pensieri e delle opinioni è uno dei diritti più preziosi dell'uomo; ogni cittadino può dunque parlare, scrivere, stampare liberamente, salvo a rispondere dell'abuso di questa libertà nei casi determinati dalla legge». Sulla Dichiarazione del 1789 la letteratura è molto estesa, v. Rials, S., La Déclaration des droits de l'bomme et du citoyen, Hachette Littératures, Paris, 1988; JaUme, L., Les Déclarations des droits de l'bomme: du Débat 1789-1793 au Préambule de 1946, Flammarion, Paris, 1989; Gauchet, M., La Révolution des droits de l'homme, Gallimard, Paris, 1989; ID., «Droits de l'homme», dans F. Furet, M. Ozouf (dir.), Dictionnaire critique de la Révolution française. Idées, Flammarion, Paris (1992) 2007, tome IV, 121-138; MorangE, J., La Déclaration des droits de l'homme et du citoyen, PUF, Paris, 2002; Bluche, F.; Rials, S.; TulaRD, J., La Révolution française, PUF, Paris, 2003; ANDriantsimbazovina, J.; GaUdin, H.; Marguenaud, J. P.; Rials, S.; Sudre, F. (dir.), Dictionnaire des droits de l'homme, PUF, Paris, 2008; LochaK, D., Les Droits de l'bomme, La Découverte, Paris (2005) 2009.

17 Vid. MathieZ, A.; Lefebvre, G., La Rivoluzione Francese, vol. 1, Einaudi, Torino, 1975, 113.

18 Vid. GuerzonI, L., «Note preliminari per uno studio della laicità dello Stato sotto il profilo giuridico», in Arch. Giur. (1967), 85 ss.; MORRA, N., Laicismo, in Nss. Dig. It., IX, Torino, 1968, 437 ss. in part. 443; ZANONE, V., «Laicismo», in Dizionario di politica, diretto da N. Bobbio e N. Matteucci, Torino, 1972, 511 ss., in part. 514; Pototschnig, U., «La laicità dello Stato», in Fus (1977), 247 ss.; CARDia, C., «Stato laico», in Encicl. Dir., XLIII, Milano, 1990, 877 ss.; Rimoli, F., «Laicità» (dir. cost.), in Enc. giur. Treccani, XIX, Roma, 1995; ID., «Laicità, postsecolarismo, integrazione dell'estraneo: una sfida per la democrazia», in Diritto pubblico (2006), 335 ss.; Mirabelli, C., «Prospettive del principio di laicità dello Stato», in Quaderni di diritto e politica ecclesiastica (2001), 331 ss.; ELIA, L., «A proposito del principio di laicità dello Stato e della difficoltà di applicarlo», in Studi in onore di Giorgio Berti, II, Iovene, Napoli, 2005; ID., «Introduzione ai problemi della laicità», in Problemi pratici della laicità agli inizi del secolo XXI, Atti del XXII Convegno annuale AIC, Napoli, 26-27 ottobre 2007, Cedam, Padova, 2008, 3 ss.; Margiotta Broglio, F., «La laicità dello Stato», in Le ragioni dei laici, a cura di G. Preterossi, Laterza, Bari, 2006; BonIolo, G., (a cura di), Laicità, Einaudi, Torino, 2006; TraVI, A., «Riflessioni su laicità e pluralismo», in Diritto pubblico (2006), 375 ss.; BARBERA, A., «Il cammino della laicità», in Forum di Quaderni costituzionali (2007); SPADARO, A., Libertà di coscienza e laicità nello Stato costituzionale. Sulle radici «religiose» dello Stato «laico», Giappichelli, Torino, 2008; Luciani, M., «La problematica laicità italiana», in Democrazia e diritto (2008), n. 2,107 ss. 
espressione della libertà religiosa, è stata ricomposta, in forma di convivenza, sulla base del principio che non è compito della politica negare la fede e che lo Stato, in quanto istituzione, realizza la sua unità di ordinamento politico territoriale indipendentemente dalla religione e facendo riferimento alle finalità e ai valori della vita terrena ${ }^{19}$.

Ciò che viene messo in ombra in questo punto di arrivo del processo di secolarizzazione è che già con il riconoscimento della libertà religiosa (ma il profilo è comune anche ad altre libertà e, in particolare, a quella di associazione) si costituiscono nel tessuto sociale delle lacerazioni, nel tempo storico considerate insanabili, per la contrapposizione delle identità riconosciute che minacciano direttamente il carattere a-religioso della politica e quello neutrale dello Stato $^{20}$.

Vero è che quest'ultimo potrebbe intervenire con la forza del diritto e, ove non basti, come spesso è accaduto, con la forza pubblica legittima, facendo prevalere la sua identità di istituzione neutrale nei confronti delle identità religiose; ma, nel momento in cui lo Stato entra nel gioco identitario, sia pure per asserire la sua laicità, non vi è più una composizione della questione, che dà luogo ad una convivenza delle fedi, bensì una risoluzione autoritativa del conflitto, che di fatto trasforma la laicità da mezzo a fine. Così, l'idea di laicità dello Stato, nata in Europa per salvaguardare la coscienza dei sudditi, si risolve - suo malgrado - come ha notato ripetutamente con riferimento alla laicità francese Paul Ricoeur in una forma di credo assoluto ${ }^{21}$.

Tuttavia, sino a quando lo Stato è dipeso esclusivamente dalla volontà del Sovrano, che ne ha esercitato l'autorità in modo assoluto, assorbendo nella sua persona anche la sfera politica, la questione non ha potuto avere un rilievo effettivo, in quanto i conflitti identitari religiosi, di fatto, sono stati relegati alla sfera sociale, senza possibilità alcuna di potere incidere effettivamente sulla decisione politica e sul modo di essere delle istituzioni pubbliche. Anche quando il sovrano ha concesso privilegi a dei sudditi, in ragione del loro particolare credo religioso, come la concessione di un territorio nel quale escludere la pratica di altri culti, o la presenza nei collegi giudiziali di uomini della propria fede, ciò non ha toccato la neutralità istituzionale dello Stato, né ha

19 Vid. Mangiameli, S., «La 'laicità' dello Stato tra neutralizzazione del fattore religioso e 'pluralismo confessionale e culturale'», in Diritto e Società (1997), 27 ss.

20 Ibidem.

21 Ricoeur, P., La critica e la convinzione, A colloquio con François Azouvi e Marc de Launay, trad. it., Milano, 1997. 
rappresentato una decisione politica in materia spirituale; atteso che il privilegio per motivi religiosi - il nome stesso lo dice - non è un diritto che disciplina il fattore religioso: è una condizione di fatto che il Sovrano ritiene di potere mantenere nel suo dominio territoriale e sotto la sua sovranità.

\section{LO STATO LAICO, LA DEMOCRAZIA E LA LIBERTÀ RELIGIOSA}

L'intera vicenda cambia connotazione nel momento in cui lo Stato si democratizza e la politica investe e rappresenta la società ${ }^{22}$.

Con la democrazia il conflitto delle identità religiose dalla società tende a riversarsi nella sfera politica. Così, sia pure in un'accezione che non è quella del medioevo e delle guerre europee di religione, sembra quasi affacciarsi un percorso inverso, e il conflitto identitario, proposto dal pluralismo sociale, finisce col toccare pur sempre e direttamente le Istituzioni pubbliche, cioè gli Stati $^{23}$.

Questi, inoltre, non essendo più governati da un Sovrano, ma essendo oramai il frutto di una sovranità frantumata, stentano a produrre il vincolo unificatore che faccia convivere le diverse fedi e che assicuri la laicità, cioè l'indipendenza dello $\operatorname{Stato}^{24}$.

Infine, poiché la democrazia è progressivamente stata connotata dal suffragio universale, dall'eguaglianza e dalla socialità come sfera di intervento privilegiata, i compiti pubblici ben difficilmente si limitano alla semplice disciplina dei quattro principi fondativi dello Stato (sicurezza esterna, pace interna, ricerca della ricchezza e godimento pacifico della libertà), ma si ritiene che questo sia competente alla giuridificazione di tutti i rapporti umani, anche se incidono sulla sfera spirituale ${ }^{25}$.

22 Elia, L. e Busia, G., «Stato democratico», in Dig. disc. pubbl., vol. XV, Utet, Torino, 1999, 56 ss.

23 Vid. già BELLINI, P., «Nuova problematica della libertà religiosa individuale nella società pluralista», in Individuo, gruppi, confessioni religiose nella società democratica, Giuffré, Milano, 1973, 1095 ss.; nonché CARDIA, C., «Religione (libertà di)», in Enc. dir., Aggiornam., vol. II, Milano, Giuffrè, 1998, 919; RicCA, M., «Art. 19», in Commentario alla Costituzione, a cura di R. Bifulco, A. Celotto, M. Olivetti, vol. I, Utet, Torino, 2006, 433.

24 Vid. Ferraioli, L., Costituzionalismo oltre lo Stato, Mucchi editore, Modena, 2017, 12.

25 Kelsen, H., La dottrina pura del diritto (1960), trad. it. di M. Losano, Einaudi, Torino, 1966, 310, usa per lo Stato la metafora di Re Mida: «il diritto è come il Re Mida: come tutto ciò che questi toccava si tramutava in oro, così tutto ciò cui il diritto si riferisce assume carattere giuridico». 
Il vero è che l'alterità tra sovrano e popolo era reale, mentre in democrazia - in via di principio - l'alterità dovrebbe essere superata o attenuata dalla rappresentanza politica; così la distinzione tra società, politica e istituzioni tende ad appannarsi in democrazia essendovi un rimando continuo da un campo all'altro in termini di contenuti e forme: tutte le richieste sociali, eminentemente private, tendono a diventare richieste politiche, eminentemente pubbliche, e ad essere assunte come compiti propri dalle istituzioni; le forme della decisione democratica richiedono necessariamente di passare dalla società alle istituzioni, attraverso la politica, e nel caso di referendum e istruttoria pubblica a fare il percorso inverso dalle istituzioni alla società. Questo andare e venire riempie lo spazio pubblico sino a congestionarlo ${ }^{26}$.

La laicità dello Stato, perciò, tende a entrare in uno stato di tensione permanente, sollecitata di continuo da accadimenti concreti che hanno un rilievo religioso e in tutti gli Stati, siano questi rigorosamente laici, come la Francia, o assertori della protezione della libertà religiosa; e ciò accade sia per gli Stati provenienti dalla tradizione cattolica, come l'Irlanda, l'Italia, la Polonia e la Spagna, come per quelli derivati dall'insegnamento luterano ed evangelico, come la Danimarca, la Germania e la Svezia.

I segni di questa tensione si sono manifestati già anche a livello europeo all'interno dell'Unione europea, come ha mostrato il dibattito sulle radici cristiane $^{27}$, svoltosi in occasione della Convenzione di Laeken per la redazione del Trattato costituzionale, risolto alla fine con l'assunto del Preambolo del Trattato dell'Unione europea, nella versione di Lisbona, che afferma il principio che le parti contraenti si ispirano «alle eredità culturali, religiose e umanistiche dell'Europa, da cui si sono sviluppati i valori universali dei diritti inviolabili e inalienabili della persona, della libertà, della democrazia, dell'uguaglianza e dello Stato di diritto» ${ }^{28}$.

26 Vid. FerRY, J. M., Le religioni nello spazio pubblico. Contributo per una società pacifica, EDB Edizioni Dehoniane, Bologna, 2017; CASANOVA, J., Oltre la secolarizzazione. Le religioni alla riconquista della sfera pubblica, il Mulino, Bologna, 2000; RORTY, R., «Religion in the Public Square. A Reconsideration», in fournal of Religious Ethics, 31.1 (2003), 141-149. LuZZATI, C., «Pluralismo, laicità e crisi della rappresentanza politica», in Stato Chiese e pluralismo confessionale, www.statoechiese.it, 2017; VANONI, L. P., Pluralismo religioso e Stato (post)secolare. Una sfida per la modernità, Giappichelli, Torino, 2016.

27 Su cui vid. WeIler, J. H. H., Un'Europa cristiana. Un saggio esplorativo, Milano, 2003, 69, che afferma «non si può predicare pluralismo culturale e nel preambolo della costituzione europea praticare l'imperialismo costituzionale».

28 Sul punto vid. Mangiameli, S., «Preamble», in H. J. Blanke, S. Mangiameli (eds.), The Treaty on European Union. A Commentary, Springer, Heidelberg, 2013, 1 ss.; ID., «L'identità dell'Europa: laicità e libertà religiosa», in Forum di Quaderni Costituzionali (2009). 
La condizione di tensione che si manifesta in democrazia sul principio di laicità è derivata dalla circostanza che la prima si avvale del principio di maggioranza, il quale all'interno delle sedi della decisone politico-istituzionale per fictio assume la connotazione di «volontà generale» giuridicamente vincolante, mentre il principio di laicità si fonda sul riconoscimento da parte dello Stato della libertà religiosa, a pieno titolo, e cioè come professione della fede in forma individuale o collettiva, di farne propaganda e di esercitarne il culto in privato o in pubblico ${ }^{29}$.

Questa libertà, come pure tutte quelle libertà «riconosciute all'uomo in quanto uomo», con l'affermazione dello stato di diritto sono basate proprio sul ritrarsi della sovranità dello Stato ${ }^{30}$, o addirittura sul carattere servente degli apparati statali per assicurare il godimento delle libertà ${ }^{31}$.

All'interno dello Stato «laico», perciò, il godimento e il rispetto dei diritti prescinde dalla relazione di «maggioranza», tipica della decisione politica istituzionale delle democrazie; la qualcosa mostra come la congiunzione di «laicità» e «democrazia» - comunemente operata -sia in realtà particolarmente problematica ${ }^{32}$.

Una democrazia «laica», proprio in quanto fonda la precettività dell'ordinamento a partire dalla libertà della fede, perciò, non può più avanzare la pretesa della «sovranità» assoluta. Lo Stato democratico, infatti, non solo è costretto ad ammettere l'originaria sussistenza di altri ordini (spirituali), per i suoi cittadini ed entro il suo territorio, che non si legittimano statualmente, ma deve anche riconoscere la validità di questi ordinamenti, rispetto ai quali ammette un limite di disciplina ${ }^{33}$.

Da questo punto di vista, poi, lo stesso ordinamento dello Stato è «laico» solo se viene riconosciuto (come «giusto») da parte di tutte le fedi. Infatti, lo

29 Vid. Gismond, P., Lezioni di diritto ecclesiastico. Stato e confessioni religiose, III ed., Milano, 1975, 59 ss.; FinOCCHIARO, F., «Articolo 19», in Commentario della Costituzione, a cura di G. Branca, Bologna-Roma, 1977, 258 ss. e in part. 265; TroILO, S., «La libertà religiosa a sessant'anni dalla Costituzione», in Forum dei Quaderni costituzionali (2008).

30 Secondo la nota impostazione di JellineK, G., Sistema dei diritti pubblici subiettivi, trad. it., Società editrice libreria, Milano, 1912.

31 Così, Esposito, C., La libertà di manifestazione del pensiero nell'ordinamento italiano, Giuffrè, Milano, 1958; Grossi, P., I diritti di libertà ad uso di lezioni, II ed., Giappichelli, Torino, 1991, 167 ss.

32 Sul punto v., in senso diverso, Rimoli, F., «Laicità» (dir. cost.), in Encicl. Giur. Treccani, Roma, 1996, 2 ss., dove, peraltro, spesso «democrazia» viene intesa come corrispondente di «pluralismo» (e viceversa).

33 Vid. DALla TORRE, G., Il primato della coscienza. Laicità e libertà nell'esperienza giuridica contemporanea, Roma, 1992, 91. 
Stato «laico», che si atteggia come lo «Stato di tutti i cittadini», «non impone il suo diritto alle diverse fedi ma lo giustifica e lo legittima per il loro stesso tramite $\gg^{34}$.

In conclusione, perciò, la dualità che caratterizza la «laicità» trova espressione lì dove lo Stato assume il compito di adottare norme che non contrastano con la fede, rectius: le fedi, ma anzi siano il frutto del loro riconoscimento. Si potrebbe sostenere, perciò, che solo la presenza della dualità, derivante dalla compatibilità dei comandi secolarizzati con i precetti della fede (rectius: delle fedi), dia la possibilità dello «Stato laico» ${ }^{35}$.

Non è agevole sostenere una siffatta conclusione e si affaccia subito il timore che in un simile Stato, limitato dalla fede, la secolarizzazione, come forma di pacificazione così duramente raggiunta, possa essere messa in pericolo; infatti, non solo dovrebbe ridursi la capacità di disciplina dell'ordinamento giuridico e ricondurre sotto condizioni più limitate la sfera della politica, per riconoscere il ruolo della religione, ma soprattutto si teme che la declinazione delle fedi al plurale, per il tramite della libertà religiosa, facendo venire meno la necessaria omogeneità democratica, non risolva il conflitto, anzi potrebbe acuirlo sino ad avere forme di violenza che minaccino la pace e la stessa libertà religiosa.

In sostanza, il pericolo che è seriamente paventato sembra quello degli effetti prodotti, non solo dalla presenza di un pluralismo religioso che divide la società in tante identità quante sono le fedi che reclamano il loro riconoscimento, ma soprattutto il venir meno, per via di un assetto democratico delle istituzioni, delle forze unificatrici che consentono allo Stato di mantenere e tutelare la sostanza della sua universalità.

Il problema e tutt'altro che apparente: un popolo è un popolo se si riconosce nello Stato, non solo per i beni terreni che questo è in grado di garantire, ma anche per il sentimento di appartenenza politica e per quegli elementi spirituali, prima ancora che materiali, che forgiano la sua identità e lo rendono riconoscibile, sia nelle relazioni pacifiche, sia sui campi di battaglia.

Allora, se un popolo è diviso spiritualmente che ne è della sua identità e di quella dello Stato?

\footnotetext{
34 PaWlowski, H. M., Introduzione alla metodologia giuridica (1986), trad. it., Giuffré, Milano, 1993, 13-14.

35 Mangiameli, S., «La 'laicità' dello Stato...», cit., 40.
} 
Se le diverse fedi religiose, presenti in seno al popolo, non sono omogenee dal punto di vista politico, quale decisione sull'identità può garantire la democrazia politica allo Stato?

\section{LA LAICITÀ ATTIVA COME NEUTRALIZZAZIONE DEL FATTORE RELIGIOSO}

Una tesi che ha avuto un considerevole seguito costituzionale è quella che spinge la laicità dello Stato ad esprimersi in una attività pubblica volta alla neutralizzazione degli effetti della libertà religiosa, a livello istituzionale e anche a livello politico ${ }^{36}$.

In questa prospettiva, non solo non vi sarebbe una valorizzazione del fattore religioso, ma al contrario si tenderebbe ad espungere ogni influenza di questo fattore dalla vita pubblica, riaffermando così una concezione della libertà religiosa, in cui questa viene intesa in modo strettamente privatistico e può, perciò, essere goduta dai cittadini solo al di fuori della vita pubblica.

Non era, pertanto, errata la previsione di chi, riflettendo sul principio di laicità, osservava che questo «potrebbe, negli anni a venire, rappresentare una sorta di 'grimaldello' con cui progressivamente espungere dal nostro ordinamento norme ed istituti, nei quali si riflette attualmente la rilevanza giuridica del fatto religioso» ${ }^{37}$.

Se si guarda alla radice di questo fenomeno di neutralizzazione, ci si avvede come abbia alla base un percorso diverso assai e poco compatibile con quella «garanzia dello Stato per la salvaguardia della libertà di religione», che dovrebbe caratterizzare l'essenza del principio di «laicità». Infatti, il punto di partenza non è rappresentato dalla valorizzazione (pubblica) del fattore religioso, nel suo «pluralismo confessionale e culturale», ma dall'affermazione del «relativismo dei valori», per il quale la «religione» non è più la ricerca della verità trascendente ispirata dalla ragione della fede ${ }^{38}$, ma una tra le possibili opinioni che si agitano nel corpo sociale. Essa si riduce, in questo modo, a mero costume storico-antropologico, rispetto al quale lo Stato, non solo deve assumere un atteggiamento «non cognitivista», ma soprattutto deve fungere

36 Vid. Rimoli, F., «Laicità», cit., 6 s., che riferisce le diverse prospettive della laicità attiva, qui di seguito riportate e criticate.

37 Dalla Torre, G., Il primato della coscienza, cit., 37.

38 Vid. Ratzinger, J., Teologia e politica della Chiesa, in Chiesa, ecumenismo e politica, San Paolo Edizioni, Torino, 1987, 147 ss. 
da garante, affinché nessun valore (e in particolare quello religioso), con la sua estensione, giunga ad escludere gli altri: non a caso si sostiene che il principio di laicità dovrebbe imporre allo Stato una «neutralità attiva», la quale tra $\mathrm{i}$ suoi compiti, paradossalmente, dovrebbe annoverare il sostegno alle concezioni minoritarie e la (de)limitazione di quelle maggioritarie, la cui presenza viene vissuta come una sorte di privilegio ${ }^{39}$.

Una concezione della laicità di questo tipo suscita non poche perplessità, in primo luogo, perché il tentativo di utilizzare l'autorità dello Stato per rendere irrilevante l'influenza religiosa dalla vita e dall'attività umana è destinato al fallimento (e comunque a non durare nel tempo) in una società in cui le comunità religiose hanno una penetrazione tale da toccare quasi l'intero «popolo»; inoltre, perché la riduzione della libertà religiosa a mera libertà di manifestazione del pensiero appare in contrasto: a) con quanto dispongono la maggior parte delle Costituzioni contemporanee, degli accordi internazionali sui diritti fondamentali e la Carta dei Diritti Fondamentali dell'Unione europea, che prevedono distintamente le due libertà ${ }^{40}$, e b), sul piano storico, con la stessa evoluzione dei diritti di libertà, che non a caso vuole le libertà derivate dalla «libertà religiosa» (e non viceversa $)^{41}$.

39 Così, in particolare, per la «neutralità attiva» Rimoli, F., «Laicità», cit., 6 ss. Va chiarito che la c.d. «neutralità attiva», o meglio ancora la neutralizzazione del fenomeno religioso, collegato al «relativismo dei valori», operando una riduzione della religione, è cosa diversa dalla «neutralità» dello Stato; quest'ultima, infatti, non presuppone nessuna riduzione del fenomeno religioso, ma il suo riconoscimento più pieno nelle sue diverse manifestazioni (v. in proposito, MORRA, G., «Religione (fenomenologia della)», in Dizionario teologico interdisciplinare, Casale Monferrato 1977, III, 43 ss.).

40 Vid., sulla libertà religiosa nel sistema della CEDU, v. MargiotTa Broglio, F., La protezione internazionale della libertà religiosa nella Convenzione Europea dei Diritti dell'Uomo, Milano, 1967; BELgiorno De Stefano, M. G., «La libertà religiosa nelle sentenze della Corte europea dei diritti dell'uomo», in Quad. dir. pol. eccl. (1989), 285 ss.; A CANNONE, «Gli orientamenti della giurisprudenza della Corte europea dei diritti dell'uomo in materia religiosa», in Riv. int. dir. uomo (1996), 264 ss.; MORVIDUCCI, C., «La protezione della libertà religiosa nel sistema del Consiglio d'Europa», in La tutela della libertà di religione: ordinamento internazionale e normative confessionali, a cura di S. Ferrari, T. Scovazzi, Padova 1998, 41 ss.; sulla Carta dei diritti fondamentali dell'UE, v. Margiotta Broglio, F., «Il fenomeno religioso nel sistema giuridico dell'Unione europea», in Religione e sistemi giuridici. Introduzione al diritto ecclesiastico comparato, a cura di F. Margiotta Broglio, C. Mirabelli, F. Onida, Bologna, 2000, 87 ss.; v. VEnTuRA, M., La laicità nell'Unione europea. Diritti, mercati, religione, Torino, 2001; MACRİ, G., «Evoluzione ed affermazione del diritto fondamentale di libertà religiosa nell'ambito della 'comunità sopranazionale' europea», in La libertà religiosa in Italia, in Europa e negli ordinamenti sopranazionali, a cura di G. Macrì, Salerno, 2003, 67 ss.; IVALDI, M. C., Diritto e religione nell'Unione europea, Roma, 2008.

41 Vid. RuffinI, F., La libertà religiosa come diritto pubblico subiettivo (1924), il Mulino, Bologna, $1992,265$. 
Ma, soprattutto, ciò che appare problematico, è la stessa ricostruzione della «laicità», fondata sul «relativismo dei valori». Il richiamo al «relativismo dei valori» opera, infatti, un salto di qualità, grazie al quale il campo di osservazione preso in considerazione (definito dalla dimensione assiologica di un gruppo) si pone in alternativa al diritto dello Stato e/o in contrasto con questo (come nel caso dei c.d. «valori emergenti»).

In tal senso, di fronte alla considerazione della condizione di fatto in cui si situano i «valori», risulta esatta l'osservazione di chi sostiene che «il regresso ai valori o ai valori fondamentali, che oggi va diffondendosi, non contribuisce in alcun modo alla stabilizzazione. Poiché $\mathrm{i}$ «valori» sono una categoria del Geltens: essi indicano - in modo molto astratto - un consenso esistente o postulato, ma non lo possono fondare da sé» ${ }^{42}$.

Di conseguenza, la postulata posizione non cognitivista dello Stato, conseguente alla presa in considerazione del relativismo assiologico del sociale, si porrebbe in contrasto con i compiti che sono attribuiti allo Stato e, la funzione di «neutralità attiva», che caratterizzerebbe lo Stato in nome della sua laicità, lo trasformerebbe da semplice soggetto garante dell'ordinamento, della legalità degli atti e della legittimità dei poteri (pubblici), a custode di un determinato patrimonio morale caratterizzato dalla molteplicità dei valori e, di più, ad un soggetto attivo che - attraverso l'uso delle sue funzioni - dovrebbe qualificarsi per un interventismo sulla sfera della «morale» tradizionalmente e storicamente diffusa nel corpo sociale (anche al fine di favorire i c.d. «valori emergenti»).

Ora, non è chi non veda come proprio l'assunzione di un compito di arbitro tra valori consolidati e valori nuovi, tra valori diffusi e valori minoritari conduca lo Stato a svolgere un ruolo che finisce col contraddire la sua dichiarata «neutralità» e a ritenersi competente a disciplinare i contenuti etici ${ }^{43}$.

La circostanza, poi, che nell'effettuare queste operazioni lo Stato sia giustificato da una spinta «anti-integralista» come garanzia del processo democratico all'interno delle istituzioni rileva a poco, dal momento che la condotta esterna finisce col fargli assumere la stessa fisionomia dello «stato etico» illiberale e totalitario. Comune a questo, infatti, risulta il principio di arginare

42 Böckenförde, E. W., Die verfassunggebende Gewalt des Volkes - Ein Grenzbegriff des Verfassungsrechts, Alfred Metzner Verlag, Frankfurt a.M., 1986, 31.

43 La qualcosa mostra, ulteriormente, la differenza esistente tra neutralità dello Stato dinnanzi al pluralismo religioso e neutralizzazione del fattore religioso (o c.d. «neutralità attiva»). Sul punto, per l'ambiguità della «neutralità dello Stato», in quanto concetto «capace di essere assunto di volta in volta con significati diversi», v. DALLA TORRE, G., Il primato della coscienza, cit., 82 ss. 
l'influenza del fattore religioso e di riconoscere allo Stato il diritto di sovrastare su ogni altra autorità (morale) di una comunità o su ogni pretesa e diritto avanzata dal singolo individuo.

\section{DECISIONE DEMOCRATICA E NEUTRALITÀ ATTIVA}

Quanto, poi, alla giustificazione della «neutralità attiva» come modo di agire che conservi il permanere del sistema democratico, essa, fondandosi sulla salvaguardia del «relativismo dei valori», riduce la «democrazia» ad una semplice procedura priva di qualunque contenuto. Una concezione questa, la quale - anche in campo liberale - ha già fatto ampiamente il suo tempo ${ }^{44}$, ma connessa proprio al tema del rapporto tra libertà (religiosa) e laicità dello Stato, finisce addirittura con l'avere un effetto perverso.

Il processo democratico all'interno dello Stato - come è noto - svolge, sul piano politico, una funzione integrante, in quanto è preposto a produrre la sintesi del molteplice attraverso l'uso del «principio di maggioranza», che dà luogo all'«atto di volontà - decisione ${ }^{45}$. Ora, in un sistema di «relativismo dei valori», dove non è più possibile esprimere una valutazione in termini di «giusto-ingiusto», «buono-cattivo», «vero-falso», la decisione democratica, da un punto di vista logico, non dovrebbe avere luogo, in quanto rappresenterebbe l'assolutizzazione di un valore rispetto ad un altro e, con ciò stesso, la negazione di quel ruolo di neutralizzazione, che dovrebbe salvaguardare il sistema democratico.

Il paradosso cui, per questa via, si giunge è tale che i sostenitori della tesi della neutralità come garanzia della democrazia finiscono col dovere necessariamente prevedere, nel momento della «decisione», una limitazione della regola democratica: il «principio di maggioranza ${ }^{46}$. Infatti, se la maggioranza è culturalmente determinata, la «neutralità attiva» imporrebbe allo Stato di non lasciare compiere il processo democratico, ma di dare luogo a una diversa

44 Vid., ad esempio, RaWls, J., Liberalismo politico, trad. it., Milano, 1994, 23 ss. e 243 ss.; nonché, Sandel, M. J., Democracy's Discontent: America in Search of a Public Philosophy, Cambridge Ma. 1995,35 ss.

45 Si v. l'efficace ricostruzione del principio in Sснмітт, C., La dottrina della Costituzione (1928), trad. it., Milano, 1984, 293 ss. e 364 ss.; di recente la ripresa del tema si deve a Pizzorusso, A., Minoranze e maggioranze, Einaudi, Torino, 1993, 3 ss.; REscignO, G. U., «Democrazia e principio maggioritario», in Quad. cost. (1994), 187 ss.

46 Sul rifiuto del principio maggioritario nell'esperienza pratica, sostituito dal c.d. «modello consensuale», v. Aмато, G., «Il dilemma del principio maggioritario», in Quad. Cost. (1994), 171 ss. 
decisione (non legittimata democraticamente) atta a salvaguardare il «relativismo dei valori».

Ad inconvenienti non minori si espone l'intervento dello Stato nell'ambito dei valori (rectius: nello spazio della loro realizzazione), dettato dalla salvaguardia di «esigenze di ordine pubblico materiale» o dalla «tutela di beni-valori costituzionalmente protetti in via primaria ${ }^{47}$.

Anche questo compito viene inteso come un aspetto essenziale della «laicità» dello Stato, in quanto fondata su una funzione attiva «anti-integralista», che si estrinseca nel limite alla (legittima) pretesa, per ogni valore, di espandersi al massimo nell'ambito del corpo sociale.

In proposito, va osservato come, in un imperante «relativismo dei valori», quello che viene ipotizzato come un civile confronto tra idee e concezioni diverse, in assenza di una misura valutativa, tende naturalmente a degenerare in forme non pacifiche. Di qui, sul piano sociale, la lotta dei valori, su quello giuridico, il conflitto dei valori e, sul piano etico, la «tirannia» dei valori ${ }^{48}$, che portano i sostenitori a posizioni demagogiche e, sul piano pratico, ad illiberali forme di assolutismo.

In una prospettiva del genere, le esigenze di «ordine pubblico materiale», o la «tutela di beni-valori protetti in via primaria» dovrebbero fungere da limite in grado di controbilanciare l'affermazione di un determinato valore, che, prevalendo sugli altri, possa imprimere al sistema una eventuale svolta «integralista».

Senonché i limiti individuati o sono irrilevanti sul piano costituzionale, operando - come nel caso dell'ordine pubblico materiale - solo dal punto di vista della situazione di fatto, oppure, se vengono visti come «interessi» o «beni» costituzionali, danno luogo ad ulteriori perplessità.

Va osservato, in proposito, che, nel caso dei valori, non mancano in genere agganci a disposizioni costituzionali positive, per cui il contrasto con i c.d. «beni-valori costituzionalmente protetti in via primaria» finisce con l'assumere l'aspetto di una conflitto (collisione) tra norme dello stesso testo normativo. Di conseguenza, o si ritiene che la Costituzione stessa contenga una gerarchia dei medesimi, la qualcosa sembra assai improbabile atteso che si tratta pur sempre di disposizioni dotate della medesima forza giuridica (e, perciò - se si vuole - di «valori costituzionali» tutti in posizione di parità); oppure, e l'ipotesi appare più realistica, la gerarchia dei valori viene scritta da chi, chiamato a

47 In tal senso RIMOLI, F., «Laicità», cit., 7 s.

48 Vid. Schmiтt, C., La tirannia dei valori, trad. it., in Rass. dir. pubbl., 1970, I, 1 ss. 
dare attuazione alla Carta costituzionale (legislatore - Corte), emette - ma in modo del tutto «arbitrario» - la decisione concretamente vincolante, su quale debba essere il «bene-valore costituzionalmente primario ${ }^{49}$.

In questo modo, il richiamo alla tutela dei «beni primari» (e cioè: superiori, dal punto di vista gerarchico), senza che questi siano espressamente indicati dalla Carta, finisce in realtà con l'indebolire i diritti costituzionali, in quanto li rende cedevoli, determinando così, dal punto di vista dell'ordinamento, una attenuazione del «principio di legalità costituzionale», il quale finisce col soggiacere alla pressione di qualunque potere che in fatto sia in grado di affermarsi ${ }^{50}$.

La «laicità», intesa come «neutralità attiva», pertanto, capovolge il rapporto tra libertà religiosa e statualità, tra libertà e decisione politica, tra costituzione e legge ${ }^{51}$. E, alla fine, nonostante la «neutralità» sia intesa ad operare una apparente riduzione assiologica delle pretese dello Stato, consente di fatto una assunzione di potere illimitato. Infatti, non a caso è stato osservato che «un potere 'neutrale' non è però un potere relativizzato o relativizzabile; anzi diviene un potere non più contestabile, perché proprio a partire dalla sua neutralità esso afferma di essere in grado di assorbire qualunque pretesa, di assolvere a qualunque compito, di riconoscere qualunque istanza e quindi di pretendere una compiuta ubbidienza: è alla fine un potere propriamente sovrano (cioè realmente 'assoluto')» ${ }^{52}$.

\section{STATO SECOLARIZZATO E LiBERTÀ RELIGIOSA: DALLA TOLLERANZA ALLA SANA LAICITÀ}

Il rifiuto dell'assolutismo statale, tuttavia, comporta anche una presa d'atto che «lo Stato liberale, secolarizzato, vive di presupposti che esso di per sé non può garantire. Questo è il grande rischio che per amore della libertà lo Stato

49 Sul punto si vedano le critiche in MANGIAMELI, S., «Il giuramento decisorio tra riduzione assiologica e ideologizzazione dell'ordinamento», in Giur. Cost. (1996), 2928 ss.

50 Efficaci le considerazioni di FoIs, S., «'Ragionevolezza' e 'valori' interrogazioni progressive verso le concezioni sulla forma di Stato e sul diritto», in Il principio di ragionevolezza nella giurisprudenza della Corte costituzionale, Giuffrè, Milano, 1994, 103 ss.; ID., «Principi e regole normative nell'opera di Vezio Crisafulli», in Il contributo di Vezio Crisafulli alla scienza del diritto costituzionale, Cedam, Padova, 1994, 249 ss.

51 Vid. in proposito le osservazioni di DogLianI, M., «Il 'posto' del diritto costituzionale», in Giur. Cost. (1993), 525 ss.

52 Così, D'Agostino, F., «Obiezione di coscienza e verità del diritto tra moderno e postmoderno», in Quad. dir. pol. eccl. (1989), 2, 6. 
deve affrontare. Come Stato liberale, esso da una parte può sussistere soltanto se la libertà che concede ai suoi cittadini si regola a partire dall'interno, dalla sostanza morale del singolo e dall'omogeneità della società. Dall'altra parte, esso non può cercare di garantire queste forze regolatrici interne da solo, ossia con i mezzi della costrizione giuridica e del comando autoritario, senza perciò rinunciare alla sua natura liberale e - sul piano secolarizzato - ricadere in quella pretesa di totalità dalla quale è uscito nelle guerre di religione» ${ }^{53}$.

$\mathrm{Ci}$ si può chiedere, allora, «se anche lo Stato secolarizzato non debba in definitiva vivere di quegli impulsi e forze di unificazione interiori mediati dalla sfera religiosa dei suoi cittadini $\gg^{54}$.

A questo punto, però, come risolvere il conflitto religioso che pervade non solo la società, ma anche la politica e le istituzioni statali?

La secolarizzazione che ha diviso le diverse sfere e collocato in ognuna quanto le è proprio: gli interessi e le passioni anche spirituali nella società, la decisione pubblica nella politica e l'unità di senso nello Stato, viene messa in discussione dal nuovo procedere degli eventi.

Questa divisione tra politica e religione, tra Stato e credo individuale, prodotta dalla secolarizzazione e che definiamo «laicità», non appare più abbastanza forte e resistente da assicurare in futuro sia la libertà che ha caratterizzato l'occidente, sia la convivenza pacifica della comunità ${ }^{55}$.

A questo problema, determinato da quello che si può definire il capolinea della secolarizzazione, non più in grado di assicurare un'identità sociale e politica, devono trovare una risposta tutti gli Stati e la stessa Europa. Ma in che modo? Verrebbe da dire, appunto, valorizzando l'apporto delle religioni.

Tuttavia, non si tratterebbe solo di superare un atteggiamento passivo nel confronto religioso o di pura banalizzazione, ritenendo il credo religioso poco più di una credenza superstiziosa, sia perché le religioni sono un'essenza dello spirito umano, che determina il pensiero e l'azione degli uomini e sia perché rappresentano una visione e un sentimento che l'individuo, insieme con altri, assume come propri.

La tematizzazione della rappresentazione fondamentale del proprio credo non può essere un tabù, così come una concezione privatistica della li-

53 BÖCKENFÖRDE, E. W., La nascita dello Stato come processo di secolarizzazione, cit., 53.

54 Ivi., 54.

55 Interessanti considerazioni sul punto in BAUBEROT, J., L'intégrisme républicain contre la laïcité, Ed. de l'Aube, La Tour-d'Aigues, 2006. 
bertà religiosa non può essere accetta. La rinuncia alla sfera pubblica, infatti, priverebbe la libertà religiosa della propria sostanza costituzionale, in quanto non sarebbe più opponibile alla decisione politica e all'azione delle istituzioni pubbliche.

Tuttavia, anche se la religione non può essere vissuta passivamente e, soprattutto, non può essere banalizzata, non può neppure spingere a un confronto distruttivo, fondato su aggressioni e sopraffazioni; al di là delle visioni del mondo professate si metterebbero in pericolo gli unici beni materiali che possono assicurare la possibilità di continuare a rappresentare il pluralismo sociale, e cioè: la pacifica convivenza e l'integrità dell'individuo.

Se la pacifica rappresentazione fondamentale della fede nella sfera pubblica si deve allora coniugare con il pluralismo delle fedi, occorre che le visioni che vengono presentate siano forti abbastanza da fornire una sana conoscenza dell'uomo.

Il tema del confronto però richiede che si assuma nella sfera pubblica una tolleranza, senza la quale il confronto stesso degenera subito in scontro ${ }^{56}$.

L'espressione «tolleranza» va qui assunta in una accezione totalmente diversa rispetto a quella con la quale nel passato si designava la relazione tra la religione di Stato e le altre credenze.

Si deve ai principi della pace di Westfalia l'affacciarsi del «principio di tolleranza» nel diritto costituzionale moderno ${ }^{57}$, così come risulta sintetizzato dalla formula dell'art. 1 dello Statuto Albertino, in cui si proclamava «la Religione Cattolica, Apostolica, e Romana è la sola Religione dello Stato. Gli altri culti ora esistenti sono tollerati conformemente alle leggi> ${ }^{58}$.

La «tolleranza», frutto della presenza cristiana o di una concezione semplicemente umanitaria, si caratterizzava come accettazione del credo della maggioranza a presupposto della vita della comunità, tanto da potere integrare l'ordinamento ricorrendo alla «morale dominante». Le minoranze dissidenti erano sopportate sin tanto che il loro comportamento portava al rispetto delle «leggi» e la loro accettazione - frutto della tolleranza - derivava dal convincimento che si trattava, fino al punto di rottura con l'ordinamento, di una posizione degna di rispetto, in quanto riconducibile ad una

56 Vid. Rousseau, J. J., Il contratto sociale, trad. it., Einaudi, Torino, 1994, $181 \mathrm{s.}$

57 RuffinI, F., Relazioni tra Stato e Chiesa, Bologna, 1974, 29 ss. che raccoglie scritti editi tra il 1908 e il 1913.

58 Vid. Racciopi, F. e Brunelli, I., Commentario allo Statuto del Regno. Articolo 1, con prefazione di L. Luzzatti, Torino, 1909, I, 53 ss. e in part. 70 s. e 73 ss. 
decisione conforme alla coscienza, anche se in contrasto con quella della maggioranza $^{59}$.

Il paradosso della «tolleranza» risiedeva, perciò, nella circostanza che chi divergeva dalla «morale dominante», pur considerato degno di rispetto, veniva considerato in errore, dal momento che la sua condotta si configurava come non conforme al credo giusto (quello della maggioranza) e, pertanto, lo Stato tollerante poteva essere accettato come «Stato proprio» solo dalla maggioranza, mentre per le minoranze dissenzienti esso avrebbe conservato i caratteri dell'imposizione esterna ${ }^{60}$.

La tolleranza che dovrebbe animare il confronto tra le fedi, così come quello tra fede e non credenza, ora, dovrebbe superare questo punto e considerare nella sfera pubblica un'effettiva parità delle fedi, di modo che l'elemento spirituale e religioso possa essere considerato come patrimonio ideale e culturale della società, in grado di sostenere la decisione politica e animare le istituzioni statali nel rispetto della libertà individuale e della pacifica convivenza.

E qui si pone il problema di una «sana laicità ${ }^{61}$.

Altrimenti detto, se è giusto ipotizzare che le istituzioni siano ideologicamente neutrali, e cioè siano laiche per astensione, non altrettanto può dirsi per la società dove idee del diverso genere trovano legittimamente posto e si misurano non solo come opinione pubblica, ma anche al fine della decisione pubblica; i temi sicuramente non mancano: dall'uso dei simboli religiosi, alle questioni inerenti al biodiritto, alla stessa concezione dell'uomo, della famiglia e del gender.

Su queste basi si può dire che, a fronte di una laicità di astensione (delle istituzioni), si situa ora nella società una laicità di confronto, frutto delle diverse idee che si raffrontano e si contrappongono.

Ora, le due laicità non stanno separate, in quanto è proprio della laicità di confronto, in un ordinamento autenticamente democratico, influenzare la decisione pubblica e ciò comporta che una vera neutralità delle istituzioni, o quanto meno delle loro decisioni, non è mai possibile del tutto. Ł̀ per questo motivo che la laicità delle istituzioni vive soltanto grazie alla laicità di confronto della società in un rapporto di circolarità e «sulla laicità di confronto

\footnotetext{
59 Sul punto vid. La tolleranza religiosa, raccolta di saggi a cura di M. Sinna, Giuffrè Milano, 1991.

60 Vid. PAwLOWsKi, H. M., Introduzione alla metodologia giuridica, cit., 13.

61 Per usare l'espressione di PIO XII, in Acta Apostolicae sedis, XXV, Città del Vaticano, 1958, 220.
} 
incombe il compito - come ha osservato Paul Ricouer - di produrre, a un certo momento della storia, un voler vivere insieme, vale a dire una certa convergenza di convinzioni ${ }^{62}$.

Da questo punto di vista, per la società civile vi sarebbe un obbligo di venire ad un accomodamento con la pluralità di opinioni che in essa si manifestano, quale caratteristica delle moderne società. Ciò è necessario, in quanto si tratterebbe di costruire quello che fohn Rawl $6^{63}$ ha definito il «consenso per sovrapposizione», grazie al quale le diversità di opinioni non generano tensioni e lacerazioni sociali, ma sono accettate e reciprocamente comprese e rispettate sulla base di un confronto che fa uso della capacità di dialogo, attraverso la fornitura dei migliori argomenti, secondo l'insegnamento della Teoria dell'agire comunicativo di Habermas ${ }^{64}$.

Se questa ricostruzione della laicità, insieme alla libertà religiosa, può convincere, allora la si può assumere come un carattere dell'identità dello Stato prodotto dalla tradizione europea, il quale continua la sua forza unificante istituzionalizzando la concordanza pratica del pluralismo sociale ${ }^{65}$.

Si può osservare come questo modo di operare della laicità si riconduca al punto di partenza di questa breve riflessione, e cioè proprio ai frutti generati dal processo di secolarizzazione che hanno portato ad una «società caratterizzata dal pluralismo, dalla non discriminazione, dalla tolleranza, dalla giustizia, dalla solidarietà e dalla parità tra donne e uomini», secondo quanto afferma l'Art. 2 TUE, per indicare l'identità dell'Unione europea e i valori comuni agli Stati membri ${ }^{66}$.

62 Ricoeur, P., La critica e la convinzione, A colloquio con François Azouvi e Marc de Launay, trad. it., Jaca Book, Milano, 1997, 185.

63 Vid. RaWls, J., Una teoria della giustizia (1971), trad. it., Feltrinelli, Milano, 1982, 32 ss.

64 Secondo l'insegnamento di HABERMAS, J., Teoria dell'agire comunicativo. Critica della ragione funzionalistica (1981), trad. it., il Mulino, Bologna, 1997.

65 Il principio della formazione della pratica concordanza (die Herstellung praktischer Konkordanz) si deve a Hesse, K., Grundzüge des Verfassungsrechts der Bundesrepublik Deutschland, 15. Aufl., C.F. Müller, Heidelberg, 1985, 127.

66 Sul punto v. Mangiameli, S., «Article 2», in The Treaty on European Union. A Commentary, cit., 109 ss. 